\title{
RapA, a bacterial homolog of SWI2/SNF2, stimulates RNA polymerase recycling in transcription
}

\author{
Maxim V. Sukhodolets, Julio E. Cabrera, Huijun Zhi, and Ding Jun Jin ${ }^{1}$ \\ Laboratory of Molecular Biology, National Cancer Institute, National Institutes of Health, Bethesda, Maryland 20892, USA
}

\begin{abstract}
We report that RapA, an Escherichia coli RNA polymerase (RNAP)-associated homolog of SWI2/SNF2, is capable of dramatic activation of RNA synthesis. The RapA-mediated transcriptional activation in vitro depends on supercoiled DNA and high salt concentrations, a condition that is likely to render the DNA superhelix tightly compacted. Moreover, RapA activates transcription by stimulating RNAP recycling. Mutational analyses indicate that the ATPase activity of RapA is essential for its function as a transcriptional activator, and a rapA null mutant exhibits a growth defect on nutrient plates containing high salt concentrations in vivo. Thus, RapA acts as a general transcription factor and an integral component of the transcription machinery. The mode of action of RapA in remodeling posttranscription or posttermination complexes is discussed.
\end{abstract}

[Key Words: RapA; SWI2/SNF2 homolog; transcriptional activation; RNA polymerase recycling; remodeling posttranscription complexes]

Received August 10, 2001; revised version accepted October 17, 2001.

In Escherichia coli, core RNA polymerase (RNAP), which consists of subunits $\alpha_{2} \beta \beta^{\prime}$, is capable of transcription elongation and termination at simple terminators. On a sigma factor binding to core RNAP, the resulting RNAP holoenzyme can initiate transcription at promoters on a DNA template (Burgess et al. 1969, 1987). There are also multiple RNAP-associated proteins, such as NusA, GreA/GreB and $\omega$, which on binding to RNAP (core and/or holoenzyme) affect various steps in the transcription cycle or RNAP assembly (Greenblatt and Li 1981; Friedman and Gottesman 1983; Sparkowski and Das 1991; Borukhov et al. 1993; Altman et al. 1994; Feng et al. 1994; Hsu et al. 1995; Mukherjee and Chatterji 1997; Minakhin et al. 2001).

Previously, we identified an E. coli RNAP-associated protein named RapA (Sukhodolets and Jin 1998). This $110-\mathrm{kD}$ protein (also known as HepA) was also reported independently by another group (Muzzin et al. 1998). RapA is a member of the SWI/SNF superfamily of helicase-like proteins, which share six evolutionarily conserved regions (Carlson et al. 1984; Andrews and Herskowitz 1989; Lewis et al. 1992; Bork and Koonin 1993; Kolsto et al. 1993; Eisen et al. 1995). Eukaryotic members of this superfamily are implicated in chromatin/ nucleosome remodeling and gene expression (for reviews, see Peterson 1996; Pazin and Kadonaga 1997;

${ }^{1}$ Corresponding author.

E-MAIL djjin@helix.nih.gov; FAX (301) 594-3611.

Article and publication are at http://www.genesdev.org/cgi/doi/10.1101/ gad.936701.
Muchardt and Yaniv 1999). These proteins are capable of altering the configuration of naked DNA, an activity that may be responsible for their chromatin/nucleosome remodeling function (Havas et al. 2000; Gavin et al. 2001).

We found that RapA binds to both core RNAP and RNAP holoenzyme, with a higher affinity for the former, at the interface of the $\alpha$ and $\beta^{\prime}$ subunits (Sukhodolets and Jin 2000). Like other members of the SWI2/SNF2 protein family, RapA is an ATPase. The ATPase activity of RapA is stimulated on binding to RNAP, indicating that RapA interacts with RNAP both physically and functionally. However, we found no apparent effect of $\operatorname{rap} A$ on cell growth in vivo and observed only a marginal effect of RapA on transcription in vitro (Sukhodolets and Jin 1998). Furthermore, our results (Sukhodolets and Jin 2000) indicated that rapA is not likely to be involved in DNA repair, contrary to a report that a mutation in the gene causes UV sensitivity (Muzzin et al. 1998).

To search for the function of RapA in transcription, we reasoned that because RapA is a bacterial homolog of SWI2/SNF2, it is conceivable that it may retain the intrinsic ability to modulate DNA conformation, leading to regulation of transcription. It is possible that RapA prefers a particular DNA conformation to act on in transcription. It is known that salt concentration has dramatic effects on the conformation of supercoiled DNA (Bednar et al. 1994; Rybenkov et al. 1997a,b). In this report we have determined the effect of RapA on transcription of supercoiled DNA at different salt concentrations and found that it greatly stimulates transcription at rela- 
tively high salt concentrations. Our results demonstrate that RapA is a general transcriptional activator important for RNAP recycling in transcription.

\section{Results}

\section{RapA activates transcription in vitro}

We decided to investigate the effect of RapA on transcription as a function of salt concentration using a supercoiled DNA containing the tac promoter (pDJ631) as DNA template (Fig. 1). We found that the magnitude of the effect of RapA on transcription is dramatically influenced by salt concentration (Fig. 1A). Specifically, RapA had a strong stimulatory effect when $\mathrm{NaCl}$ was higher than $100 \mathrm{mM}$; the activation reached its maximum at $200 \mathrm{mM} \mathrm{NaCl}$. At relatively low ( $\leq 100 \mathrm{mM}$ ) or very high (300 mM) salt concentrations, RapA had only a minimal effect on transcription. In the absence of RapA, transcription from the tac promoter was also sensitive to salt concentration. The transcription was enhanced when the $\mathrm{NaCl}$ concentration was increased up to $250 \mathrm{mM}$ and then declined at $300 \mathrm{mM} \mathrm{NaCl}$. However, the magnitude of the stimulation was several-fold lower than that observed with RapA. Thus, RapA is a potent transcriptional activator at relatively high concentrations of $\mathrm{NaCl}$. Similar results were obtained when $\mathrm{KCl}$ was used (Fig. 1B).

Because potassium glutamate, rather than $\mathrm{NaCl}$ or $\mathrm{KCl}$, is the major intracellular salt in E. coli (Cayley et al. 1991), the effect of this salt on the ability of RapA to activate transcription was determined (Fig. 1C). RapA activated transcription of tac significantly only when potassium glutamate concentrations were $\geq 400 \mathrm{mM}$, with maximal stimulatory effects at $\sim 500 \mathrm{mM}$ potassium glutamate. The consistent behavior of RapA in the presence of different kinds of salt indicates that RapA generally requires a relatively high salt concentration to stimulate transcription.

The ability of RapA to activate transcription depends on a supercoiled DNA template. When the same plasmid DNA was linearized, transcription was more sensitive to salt concentration overall than that on supercoiled DNA (at $\geq 250 \mathrm{mM} \mathrm{NaCl}$ RNAP nearly ceased synthesis of the tac transcript), and RapA exhibited an inhibitory effect at all salt concentrations tested (Fig. 2).
A
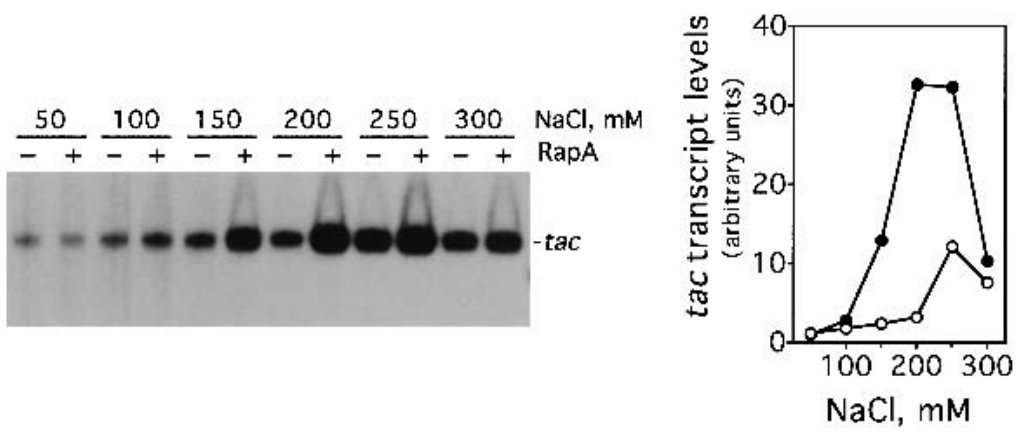

B
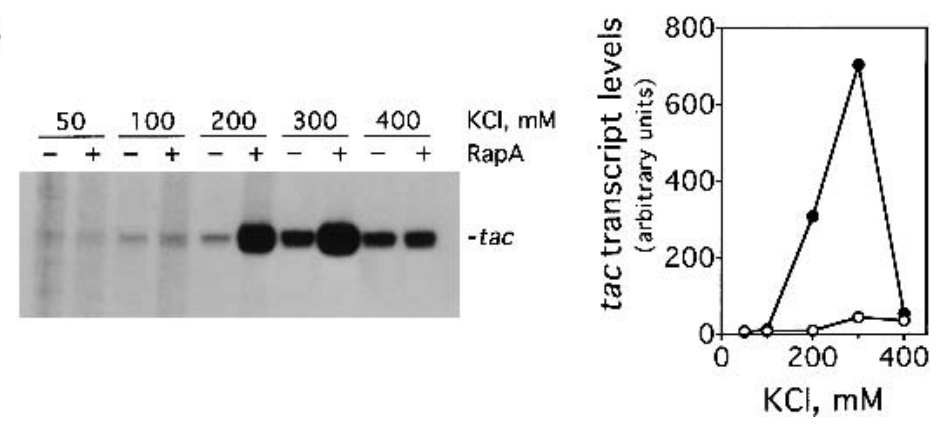

C

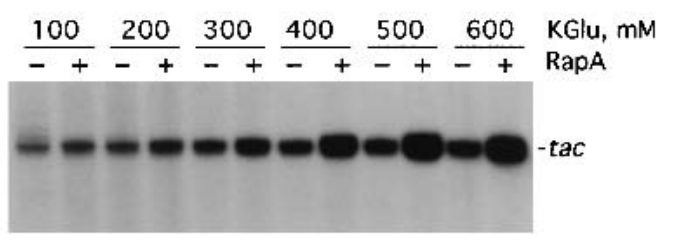

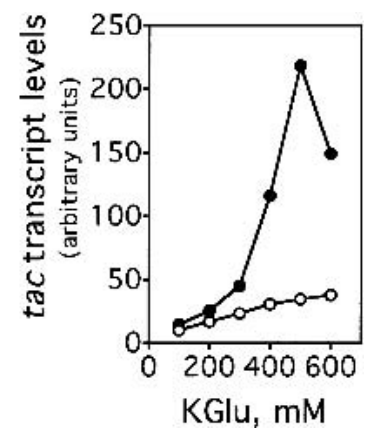

Figure 1. RapA stimulates transcription on supercoiled DNA in the presence of high concentration of salts. in vitro transcription reactions were carried out as described in Materials and Methods. Autoradiographs of the in vitro transcription gels showing the terminated tac transcript are presented. The NTP concentrations $(\mathrm{A} / \mathrm{U} / \mathrm{G} / \mathrm{C})$ were $1 / 0.2 / 0.2$ / $0.02 \mathrm{mM}$, respectively. The concentrations of RNAP holoenzyme, RapA (purified from the 1:1 RNAP holoenzyme-RapA complex), and supercoiled plasmid DNA template (pDJ631) were approximately $12 \mathrm{nM}, 0.4 \mu \mathrm{M}$ (when present), and $12 \mathrm{nM}$, respectively. The effect of RapA on transcription from the tac promoter as a function of: $\mathrm{NaCl}$ concentration $(A), \mathrm{KCl}$ concentration $(B)$, and potassium glutamate (KGlu) concentration $(C)$. The quantitated levels of the tac transcript in reactions with (solid symbols) or without (open symbols) RapA are shown at right. 
Figure 2. RapA fails to stimulate transcription on linear DNA. The experimental conditions were similar to those described in Figure 1A, except that the purified 1.2-kb $B f a$ I DNA fragment of pDJ631 (the $B f a I$ sites are located $~ 900 \mathrm{bp}$ upstream and $\sim 300$ bp downstream from the tac promoter in the plasmid) at the concentration of $\sim 5$ $\mathrm{nM}$ was used as a DNA template. The quantitated levels of the tac transcript in reactions with (solid symbols) or without (open symbols) RapA are shown at right.
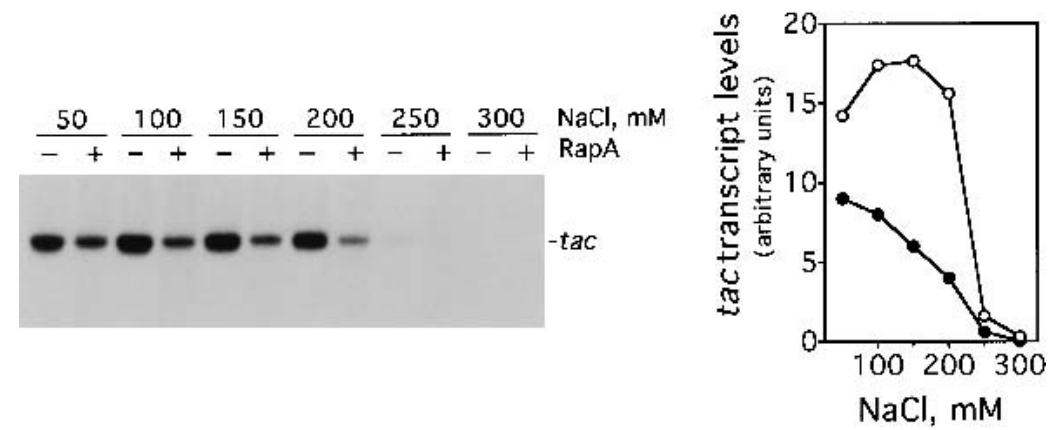

To rule out the possibility that RapA-mediated transcriptional activation was promoter-specific, we determined the effect of RapA on several other promoters (Fig. 3). RapA was capable of stimulating transcription at all the promoters tested when a relatively high concentration of salt was present in the reaction (under the conditions used, RapA increased RNA synthesis 4- to 20fold compared to RNAP alone), although each promoter had its own characteristic profile. In the case of the stringent promoters $\operatorname{rrn} B P 1$ or pyrBI, there was no transcription at $300 \mathrm{mM} \mathrm{NaCl}$ either in the presence or absence of RapA, reflecting the intrinsic instability of initiation complexes on stringent promoters (Gourse 1988; Zhou and Jin 1998). These results indicate that RapA has not altered the properties of these promoters.

\section{The ATPase activity of RapA is essential for its function as a transcriptional activator}

Because RapA is an ATPase and its ATPase activity is stimulated by binding to RNAP (Sukhodolets and Jin 2000), we asked whether the ATPase activity of RapA is required for transcription activation. We first introduced two different rapA mutations in the conserved regions involved in NTP-binding: Lys 183 to Ala (SWI/SNF motif Ia) and Asp280-Glu281 to Ala-Ala (SWI/SNF motif II). These two mutations at homologous sites in the yeast SWI/SNF proteins greatly impair ATPase and chromatin/nucleosome remodeling activities (Peterson and Tamkun 1995).

The purified mutant RapA proteins (Fig. 4A) had greatly reduced ATPase specific activities (Fig. 4B). The ATPase-specific activity of recombinant wild-type RapA alone was 18.4 pmole of ATP hydrolyzed per $\mu g$ of protein/min, a value comparable to that of the endogenous RapA purified from the 1:1 RNAP-RapA complex (28 pmole of ATP hydrolyzed per $\mu \mathrm{g}$ of protein/min; Sukhodolets and Jin 1998). In contrast, the ATPase-specific activities for both mutant proteins were 10-fold lower than that of the recombinant wild-type RapA. However, addition of RNAP stimulated the ATPase specific activities of both mutant RapA proteins to the same extent as wild-type recombinant RapA (threefold), suggesting that the mutant RapA proteins were capable of forming a complex with RNAP. Consistent with this, we detected little (SWI/SNF motif II RapA mutant) or no (SWI/SNF motif Ia RapA mutant) difference in the affinity of the two mutant enzymes to RNAP compared to recombinant wild-type RapA using a glycerol gradient ultracentrifugation-binding assay (Sukhodolets and Jin 2000). However, the affinity of recombinant wild-type RapA to RNAP was reduced $\sim 5$ - to 10 -fold compared to that of wild-type (endogenous) RapA purified from the 1:1 RNAP-RapA complex (data not shown).

We then tested the effects of the two mutant RapA proteins on transcription in the presence of high concentrations of $\mathrm{NaCl}$. For the recombinant wild-type RapA protein, it showed stimulatory effect on transcription of tac at a molar ratio (RapA/RNAP) 1 ; the stimulatory effect approached its maximum at $>10$-fold excess of RapA over RNAP (Fig. 4C). Although the recombinant wildtype RapA protein activated transcription, the mutant proteins were unable to do so, even at relatively high concentrations (Fig. 4D). Therefore, the ATPase activity of RapA is essential for its function as a transcriptional activator, indicating that the ATPase activity of RapA is coupled to RapA-mediated transcriptional activation.

\section{RapA stimulates RNAP recycling in transcription}

To gain insight into the mechanism by which RapA activates transcription, we analyzed the kinetics of in vitro transcription reactions (Fig. 5). In one set of experiments, the reactions were started by the addition of RNAP or RNAP plus RapA into a preincubated solution containing DNA and NTPs (Fig. 5A); that is, RNAP was not allowed to form initiation complexes before the addition of NTPs. In another set of experiments, the reactions were started by the addition of NTPs into a preincubated solution containing the DNA template plus either RNAP or RNAP and RapA (Fig. 5B); that is, RNAP-DNA complexes could form before the start of transcription. Both sets of experiments yielded very similar results. At early timepoints ( $\leq 1 \mathrm{~min})$ RapA exerted an inhibitory effect when compared to RNAP alone (Fig. 5A,B, 3× exposures; also, Fig. 5C, inset). However, as the reactions progressed, the stimulatory effect of RapA on transcription became apparent, and continued to increase in proportion to the reaction time (Fig. 5A,B, 1× exposures; also, Fig. 5C). In reactions containing RNAP alone, RNA synthesis from the $\operatorname{rrn} B$ P1 ceased after $\sim 5 \mathrm{~min}$, whereas in the presence of RapA, RNA synthesis continued even 
A

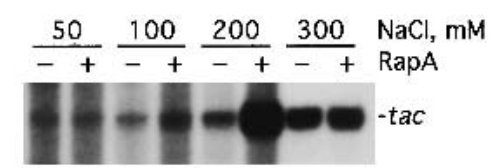

B

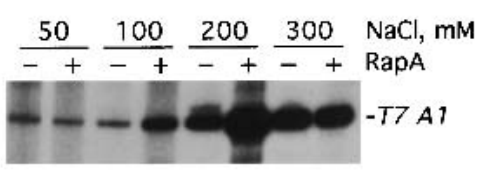

C

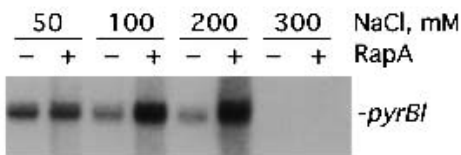

D

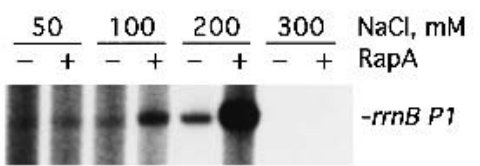

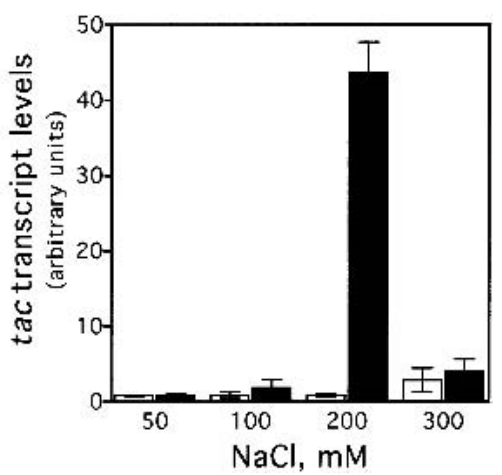
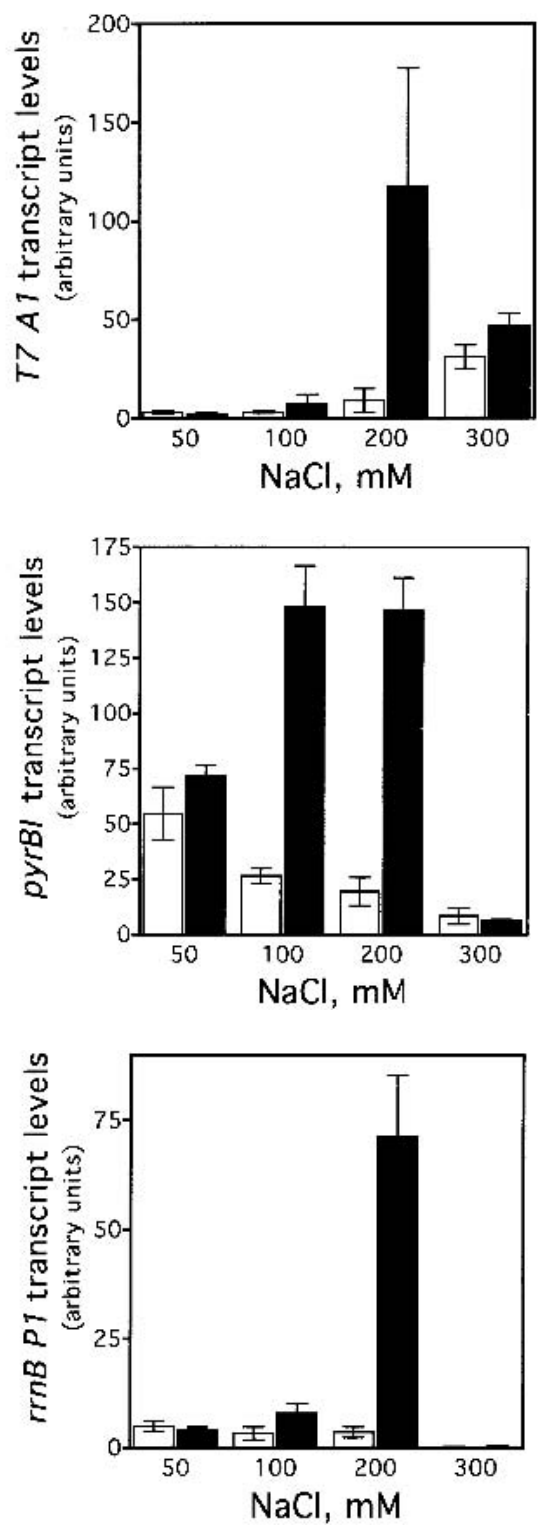

Figure 3. RapA-mediated transcriptional activation is not promoter-specific. In vitro 60 -min transcription reactions were carried out as described in Materials and Methods. Autoradiographs of the in vitro transcription gels are shown, and the positions of the promoter-specific transcripts are indicated. The NTP concentrations $(\mathrm{A} / \mathrm{U} / \mathrm{G} / \mathrm{C})$ were $1 / 0.2 / 0.2 / 0.2 \mathrm{mM}$, respectively. The concentrations of RNAP holoenzyme, purified recombinant RapA, and the supercoiled plasmid DNA templates were approximately $12 \mathrm{nM}, 0.6 \mu \mathrm{M}$ (when present), and $12 \mathrm{nM}$, respectively. The data demonstrate the effects of RapA on transcription as a function of $\mathrm{NaCl}$ concentration with a supercoiled plasmid containing: the tac promoter $(\mathrm{pDJ} 631)(A)$, the T7 $A 1$ promoter $\left(\mathrm{pCPG} \mathrm{t}_{\mathrm{R} 2}\right)(B)$, the pyrBI promoter (pBHM332) (C), and the rrnB P1 promoter (pRLG1617) (D). The quantitated levels of the transcripts in the absence (open bars) or in the presence (solid bars) of RapA are shown at right. Each graph represents data from four independent experiments. after 30 min (Fig. 5C). In fact, RapA enabled continued RNA synthesis even after $90 \mathrm{~min}$ in the in vitro transcription reactions. Similar results were obtained from kinetic studies with another supercoiled DNA template containing the tac promoter (data not shown).
These kinetic studies indicate that the RapA-mediated transcriptional activation is manifested in multipleround transcription reactions; therefore, no stimulatory effect in single-round transcription reactions should be expected. To test this hypothesis, we analyzed the effect 
Figure 4. The ATPase activity of RapA is essential for its function as a transcriptional activator. $(A) \mathrm{Pu}-$ rified recombinant RapA proteins (1 $\mu \mathrm{g}$ each) are shown on a Coomassie Brilliant Blue R-250-stained SDS $-10 \%$ polyacrylamide gel. (Lane 1) $5 \mu \mathrm{L}$ of the BioRad protein molecular weight standards (broad range); (lane 2) wild-type RapA; (lane 3) the SWI/SNF motif Ia RapA mutant; (lane 4) the SWI/SNF motif II RapA mutant. (B) The ATPase specific activities of the mutant RapA proteins are greatly reduced. ATPase activity was determined as described in Materials and Methods; an autoradiograph of a representative ATPase assay is shown. When present, the concentration of recombinant RapA was $0.9 \mu \mathrm{M} / \sim 1$ $\mu g$ per reaction); the RNAP holoenzyme concentration was $0.5 \mu \mathrm{M}(\sim 2.2 \mu \mathrm{g}$ per reaction). (C) Titration of the recombinant wild-type RapA protein for its stimulatory effect on transcription. In vitro transcription reactions were performed in the presence of $250 \mathrm{mM} \mathrm{NaCl}$, as described in Materials and Methods. An autoradiograph of a representative experiment is shown. The conditions were similar to those described in Figure 3, except that the concentrations of RNAP and the supercoiled DNA template (pDJ631) were $12 \mathrm{nM}$ and $24 \mathrm{nM}$, respectively. The molar ratio of RapA and RNAP used in each reaction is indicated. The quantitated levels of the tac transcript as a function of the amounts of RapA (as ratios of RapA and RNAP) used in reactions are also shown. (D) The mutant RapA proteins are defective in transcriptional activation. In vitro transcription reactions were carried out in the presence of $220 \mathrm{mM} \mathrm{NaCl}$, as described in Materials and Methods. The representative autoradiograph of the in vitro transcription gel is shown; the position of the tac transcript is indicated. The NTP concentrations (A/U/G/C) were 1/0.2/0.2/0.2 mM, respectively. The RNAP holoenzyme concentration was $\sim 14$ nM; the supercoiled DNA template (pDJ631) concentration was $\sim 12 \mathrm{nM}$. The RapA concentration was $32 \mathrm{nM}$ in lanes 2, 6, 10; $160 \mathrm{nM}$ in lanes 3, 7, 11; and $640 \mathrm{nM}$ in lanes 4,8 , and 12 , respectively.

of RapA on transcription of tac in the presence of a DNA competitor, heparin, which binds free and/or dissociated RNAP, thus preventing reinitiation (Fig. 6). In the absence of heparin, the RapA-mediated transcriptional activation was $>10$-fold; however, the ability of RapA to stimulate transcription was significantly reduced at low concentrations of heparin and totally abolished at high concentrations of the DNA competitor. Note that for RNAP alone, there was only a marginal $(<1.5$-fold $)$ difference in the amount of RNA synthesized between single-round and multiple-round transcription reactions, indicating that RNAP recycling is limiting under the conditions used. Other DNA competitors, such as nonspecific plasmid DNA, yielded results similar to those obtained with heparin (data not shown). These results indicate that RapA stimulates RNAP recycling, leading to effective multiple-rounds of transcription.

The rapA null mutant is defective in growth at high salt concentrations in vivo

Previously, the rapA null mutant showed no obvious growth deficiencies under a variety of laboratory condi- tions (Sukhodolets and Jin 2000). The observation that RapA-mediated transcriptional activation requires high salt concentrations led us to ask whether the rapA mutant exhibits growth defects in media containing high salt concentrations. We plated cells from both the rapA null mutant and the isogenic wild-type strains on LB plates containing different $\mathrm{NaCl}$ concentrations and followed their growth for different lengths of time after incubation at $30^{\circ} \mathrm{C}$. The growth of the rapA mutant was seriously inhibited on LB plates containing $1 \mathrm{M} \mathrm{NaCl}$ compared to wild-type cells (Fig. 7A), although there was no difference in growth between the two strains on LB plates containing $\leq 0.5 \mathrm{M} \mathrm{NaCl}$. On incubation for two more days, however, the rapA mutant was able to grow on LB plates containing $1 \mathrm{M} \mathrm{NaCl}$ with an efficiency similar to that of the wild-type strain, indicating that the $\operatorname{rap} A$ gene affected the rate of growth rather than the plating efficiency. Thus, the in vivo effect of rapA is consistent with that of RapA in vitro. Furthermore, the growth defect of the rapA mutant was corrected by introducing a plasmid containing a functional rapA gene into the cells (Fig. 7B), confirming that rapA is important for cell growth at high salt concentrations. 
A

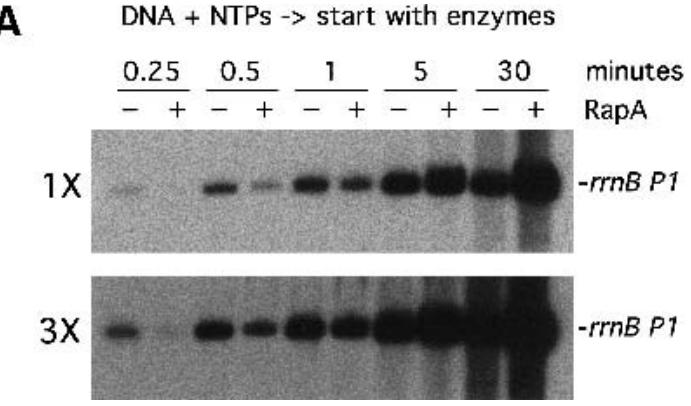

B Enzymes + DNA $\rightarrow 15 \mathrm{~min}, 37^{\circ} \mathrm{C} \rightarrow$ start with NTPS

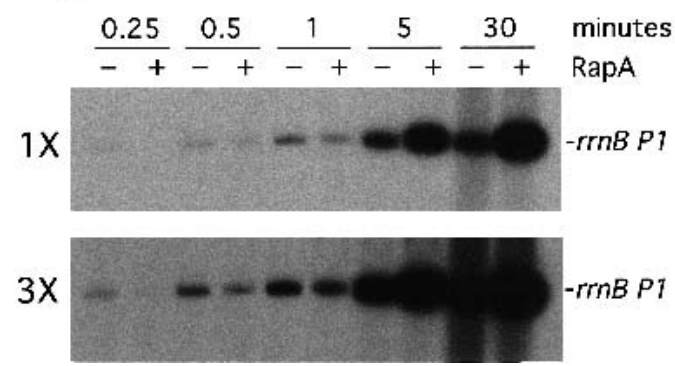

\section{C}

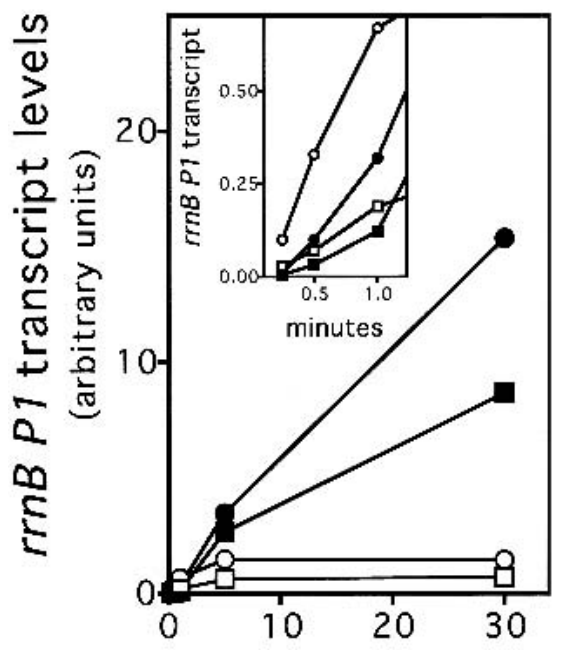

Time $(\mathrm{min})$

Figure 5. RapA-mediated transcriptional activation is manifested in prolonged, multiple-round transcription reactions. Kinetics of the RapA effect was studied in the presence of $200 \mathrm{mM} \mathrm{NaCl}$, using the pRLG1617 supercoiled DNA template. The other ingredients in the reactions were the same as those described in Figure 3. Autoradiographs at $1 \times$ and $3 \times$ exposure (see Materials and Methods) of representative in vitro transcription gels are shown. $(A)$ The reactions were initiated by the addition of enzymes into a reaction mixture containing DNA and NTPs. (B) The reactions were initiated by the addition of NTPs into a preincubated $\left(15 \mathrm{~min}\right.$ at $\left.37^{\circ} \mathrm{C}\right)$ mixture containing enzymes and DNA. $(C)$ The quantitated levels of the promoter ( $r n B$ P1)-specific transcript generated in in vitro transcription reactions initiated by the addition of NTPs (rectangles) or enzymes (circles) in the absence (open symbols) or presence (solid symbols) of RapA; inset shows early timepoints. Note that overall RNA synthesis in the reactions started with NTPs was lower than that started with RNAP (this was a reproducible result), suggesting that some fraction of RNAP became nonfunctional when RNAP was incubated with DNA first, probably by trapped at some strong binding sites (non-promoter) in DNA.

The inhibition of growth of the rapA mutant on LB plates containing $1 \mathrm{M} \mathrm{NaCl}$ appeared to be attributable to high salt concentration rather than high osmolality. When we plated cells on LB plates containing $1 \mathrm{M}$ sucrose, neither wild type nor the rap $A$ mutant could grow. The growth of both wild-type and the rapA mutant was severely inhibited to the same extent on LB plates containing $0.75 \mathrm{M}$ sucrose (data not shown).

\section{Discussion}

We have shown that RapA, an E. coli RNAP-associated protein and a bacterial homolog of the SWI/SNF protein family, is capable of dramatic stimulation of in vitro transcription. The requirements for RapA-mediated transcriptional activation are (1) relatively high salt concentrations, (2) a supercoiled DNA template, and (3) conditions favoring multiple-round transcription reactions. Furthermore, the ATPase activity of RapA is essential for its function as a transcriptional activator. Our data indicate that the mode of action of RapA is to promote RNAP recycling during transcription.

We believe that RapA, rather than some minor contaminant in the RapA preparations, is responsible for the observed stimulatory effect on transcription for the following reasons: (1) The RapA preparations obtained us-
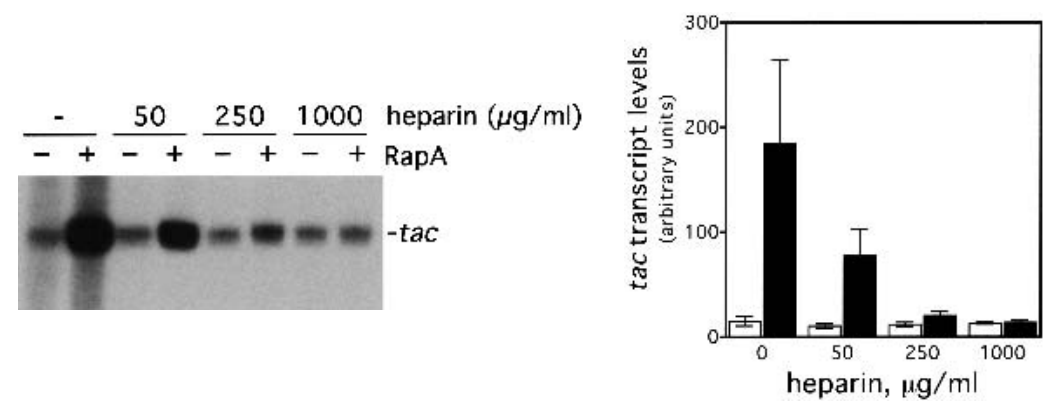

Figure 6. The DNA competitor heparin inhibits transcriptional activation by RapA. In vitro 60min transcription reactions were performed in the presence of $200 \mathrm{mM} \mathrm{NaCl}$, using the pDJ631 supercoiled DNA template. The other ingredients in the reactions were the same as those described in Figure 3. An autoradiograph of a representative in vitro transcription gel is shown. The heparin concentrations are indicated. The quantitated levels of the tac transcript in reactions with /solid bars) or without (open bars) RapA are also presented. The data represent an average of two independent experiments. 
Sukhodolets et al.

A

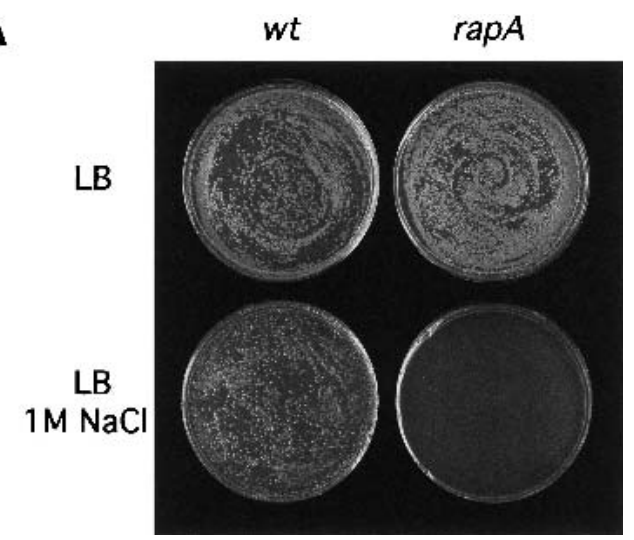

B

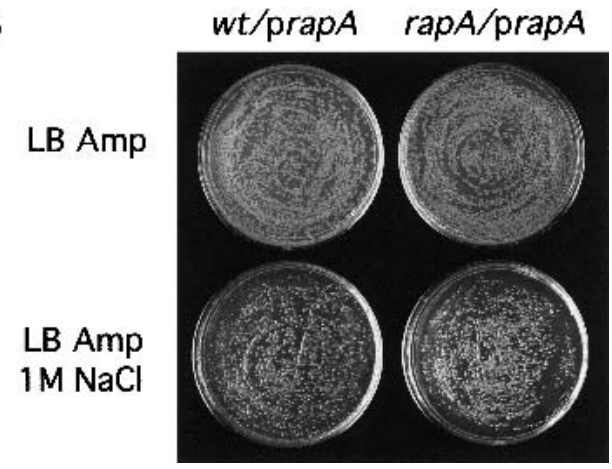

Figure 7. The rapA null mutant is defective in growth on LB plates containing high concentrations of salt. (A) Overnight cultures of wild-type (MG1655) strain and the rapA null mutant (DJ473) were diluted and plated on LB plates $\pm 1 \mathrm{M} \mathrm{NaCl}$, as described in Materials and Methods. Plates were incubated at $30^{\circ} \mathrm{C}$ for $16 \mathrm{~h}$ (top) or $48 \mathrm{~h}$ (bottom). (B) Overnight cultures of wild-type (MG1655) strain containing pDJ2635 (prapA) or the rapA null mutant (DJ473) containing pDJ2635 (prapA) were diluted and plated on LB + ampicillin plates $\pm 1 \mathrm{M} \mathrm{NaCl}$, as described in Materials and Methods. Plates were incubated at $30^{\circ} \mathrm{C}$ for $16 \mathrm{~h}$ (top) or $60 \mathrm{~h}$ (bottom).

ing dissimilar purification procedures (either from the 1:1 RNAP holoenzyme-RapA complex or from cells overproducing the His-tagged recombinant proteins) all activated transcription, and (2) the mutant RapA proteins, purified the same way as the wild-type recombinant RapA failed to activate transcription. Although we used an excess of RapA in the reactions to ensure the maximal stimulatory activity, a 1:1 ratio of RapA and RNAP was enough to observe the stimulatory effect of RapA on transcription in vitro (Fig. 4C). In E. coli, the ratio of RapA and RNAP is $\sim 1 / 10$ (and the ratio of sigma 70 and RNAP is $\sim 1 / 3$ ), as estimated from RNAP preparations purified from cells of late-log growth phase (Sukhodolets and Jin 1998).

\section{Tightly compacted supercoiled DNA is the preferred substrate for RapA}

The conformation of supercoiled DNA is very sensitive to salt concentration (Adrian et al. 1990; Bednar et al.
1994; Rybenkov et al. 1997a,b). The overall shape of supercoiled DNA undergoes a dramatic conformational change from loosely supercoiled DNA at low salt concentrations to tightly supercoiled DNA at relatively high salt concentrations in vitro (Bednar et al. 1994). In these counterion-induced, tightly supercoiled DNA molecules, the opposing segments of interwound superhelix appeared to be in close proximity longitudinally with small terminal loops called apexes at both ends of the molecules (Bednar et al. 1994). This tightly supercoiled DNA is not static, but rather has a very dynamic structure that allows aligned segments to move freely with respect to each other (Brady et al. 1983; Spengler et al. 1985). Such a feature is unique to supercoiled DNA because neither nicked circular nor linear DNA molecules under the same conditions exhibit signs of intersegmental attraction, suggesting that the free energy of supercoiling is needed to induce the formation of tightly supercoiled DNA.

Intriguingly, RapA exerts its dramatic stimulatory effect on transcription only with supercoiled DNA at relatively high salt concentrations in vitro, a condition that is likely to render the DNA template tightly supercoiled. Such an apparent correlation between the effects of salt on DNA conformation and that on RapA-mediated transcriptional activation indicates that tightly supercoiled DNA is the substrate for RapA in transcription. Our study suggests that such a DNA conformation is biologically relevant in the modulation of transcription.

\section{Mode of action by RapA in transcription}

We have demonstrated that RapA stimulates RNAP recycling in transcription, based on the observation that the effect of RapA is manifested dramatically only in prolonged, multiple-round transcription reactions. Presently, we do not know how RapA stimulates RNAP recycling; however, we can speculate about the mechanism based on the results described below and other experimental observations. Because RapA exhibited inhibitory rather than stimulatory effects on transcription of $\operatorname{rrnB} P 1$ in vitro at early timepoints (Fig. 5), it is unlikely that RapA activates transcription by enhancing the efficiency of initiation at steps prior to "open complex" formation. Furthermore, RapA did not alter the unstable nature of initiation complexes at stringent promoters (Fig. 3), indicating that it is not likely to be acting at the initiation step. Also, using DNase I footprinting and a gel-filtration-based binding assay (in which transcription mixtures were subjected to rapid gel-filtration to estimate the fractions of free and DNAbound RNAP and RapA), we detected no difference in binding to supercoiled DNA between RNAP alone and RNAP + RapA, under conditions in which RapA greatly stimulated transcription. We also examined the effect of RapA on promoter clearance by analyzing nonproductive synthesis products from several promoters and found that RapA did not facilitate promoter clearance. The fact that RapA has a higher affinity for core RNAP than for 
RNAP holoenzyme (Sukhodolets and Jin 2000) is consistent with the notion that RapA is not likely acting at the initiation steps.

Moreover, we detected no significant effect of RapA on the rate of elongation, and RapA was capable of activating transcription with either low $(50-200 \mu \mathrm{M})$ or high (1-5 mM) NTP concentrations in the reactions. Together, our data indicate that RapA affects RNAP recycling, operationally, at a step subsequent to termination and prior to reinitiation.

There was only a marginal difference in RNA synthesis by RNAP alone between single-round and multipleround in vitro transcription reactions (Fig. 6), indicating that the RNAP molecule becomes nonfunctional after completing only one or two rounds of transcription under the conditions used. This apparent RNAP inactivation is the result of transcription, because RNAP incubated with DNA and transcription buffer without NTPs was still active after a prolonged incubation (data not shown).

The reason(s) for the failure of RNAP to recycle effectively during transcription is unknown at present. However, we speculate that an RNAP molecule (either with or without associated nascent RNA) that has completed one or two rounds of transcription becomes trapped or immobilized at terminator(s) or at some sites in DNA nonspecifically, forming a posttranscription or posttermination complex (PTC). This takes place, presumably, because of conformational changes in either RNAP and/ or DNA that occur as a result of transcription. This, in turn, restricts the movement of RNAP and/or DNA segments of the PTC.

We propose that RapA remodels the PTC to promote the free movement of RNAP and/or DNA segments of tightly supercoiled DNA (Fig. 8). Such movement could liberate the RNAP molecule (either by triggering termination or by enhancing dissociation of RNAP from DNA/RNA), leading to its release from the PTC. Alternatively, the free movement of tightly supercoiled DNA segments could facilitate a proper spatial arrangement that would allow RNAP to transfer directly from a terminator or other nonspecific sites to a promoter to initiate another round of transcription. In essence, RapA remodels the nonproductive PTC to a productive transcription complex, enabling subsequent cycles of transcription. In this scenario, the energy provided by ATP hydrolysis would be the driving force of the RapA motor to remodel the PTC of tightly supercoiled DNA. Recently, several eukaryotic SWI/SNF proteins have been found to modulate the conformation of naked DNA and enhance the mobility of DNA-binding proteins (Havas et al. 2000; Gavin et al. 2001; Ristic et al. 2001). Our results and model for RapA function are consistent with those findings.

The biological significance of RNAP recycling is obvious. Although the vast majority of the studies on the regulation of transcription have focused on different aspects of a single transcription cycle, very few studies have addressed the issue of RNAP recycling. It has been reported that a $20-\mathrm{kD}$ auxiliary subunit of Bacillus subtilis RNAP known as delta $(\delta)$ is able to stimulate RNAP recycling by facilitating the release of RNA from the RNAP-RNA complex (Juang and Helmann 1994). Unlike RapA, $\delta$ activity does not depend on supercoiled DNA. It has also been reported that E. coli sigma factor-70 facilitates the release of RNAP from the RNAP-DNA complex after transcription termination (Arndt and Chamberlin 1988). Thus, it may also contribute in part to

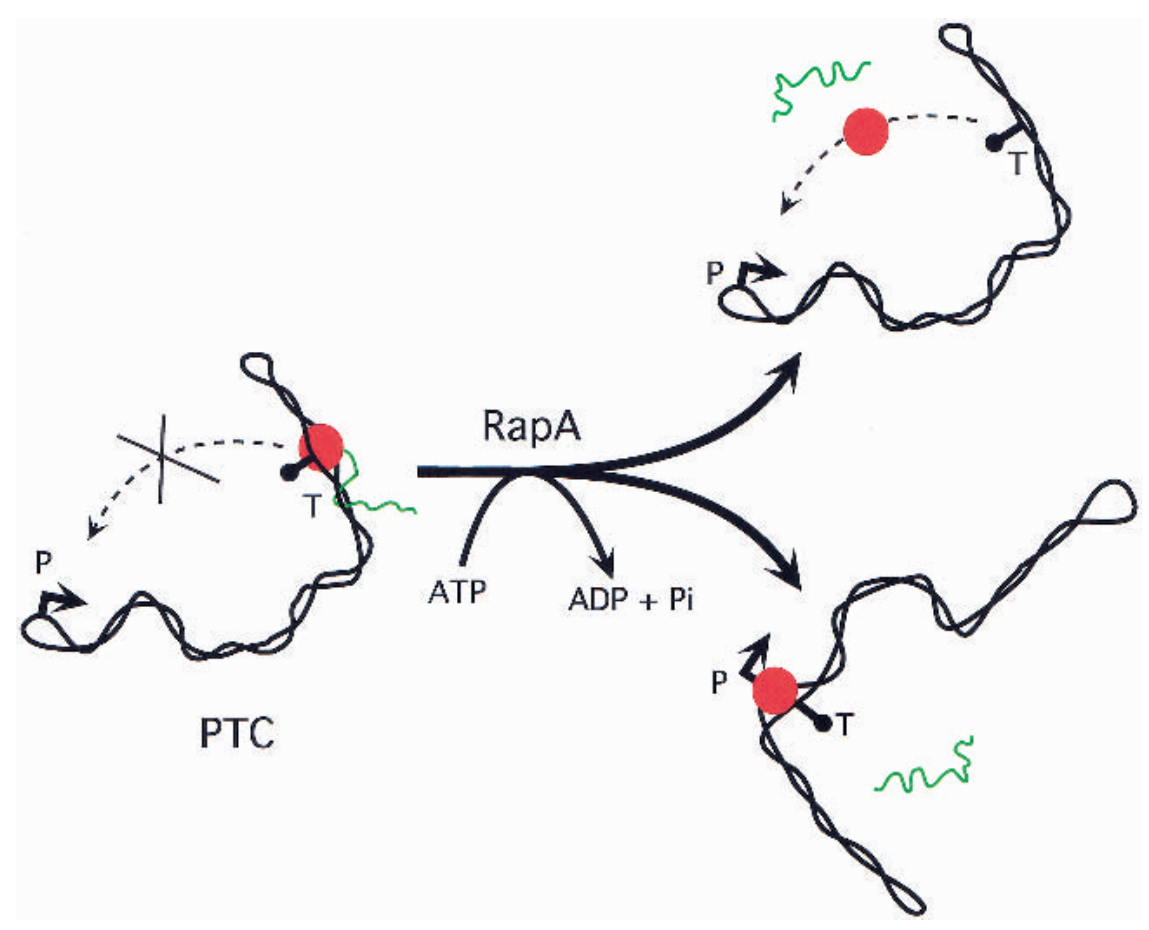

Figure 8. A model for the mode of action by RapA: Remodeling of a posttranscription/posttermination complex on tightly supercoiled DNA. After completing one round of transcription (including termination at the terminator sequence T), RNAP (red) with associated RNA (green) is trapped or immobilized on tightly supercoiled DNA, forming a posttranscription/ posttermination complex (PTC). This will prevent RNAP from reinitiating another round of transcription (i.e., dissociation from DNA/RNA and interaction with promoter $\mathrm{P}$, which is likely located at one of the apexes of tightly supercoiled DNA template; ten Heggeler-Bordier et al. 1992). RapA will remodel PTC so that: (1) RNAP is released from the complex and binds to the promoter to initiate another round of transcription, and/or (2) the free movement of DNA segments is enhanced to facilitate a proper spatial arrangement for transfer of RNAP from terminator or other nonspecific sites in DNA to a promoter to initiate another round of transcription. 
RNAP recycling. Apparently, one common theme for both the sigma factor and $\delta$ is their ability to weaken the interaction between RNAP and nucleic acid (RNA and/ or DNA). Our model (Fig. 8) that RapA promotes the release of RNAP trapped at a DNA template (either at terminator or other nonspecific sites) after one round of transcription and/or enhances the movement of DNA leading to stimulation of RNAP recycling is consistent with this theme. Moreover, the results from heparin competition experiments (Fig. 6) argue for release of RNAP by RapA. Experiments to test our model are currently underway.

\section{Cellular function of rapA}

The observed growth defect of the $\operatorname{rap} A$ mutant on $\mathrm{LB}$ plates containing $1 \mathrm{M} \mathrm{NaCl}$ is consistent with the notion that RapA is important for transcription under the stress conditions induced by high concentrations of salt. In $E$. coli cells the concentration of counterions that bind to DNA is unknown, although it has been reported that the intracellular concentration of $\mathrm{K}^{+}$in bacterial cells ranges from $200 \mathrm{mM}$ to $900 \mathrm{mM}$ depending on environmental conditions (Richey et al. 1987). Concentrations of other salts in E. coli have also been reported (Cayley et al. 1991). It is conceivable that under some growth conditions, presumably, in the presence of high concentrations of salt, the E. coli chromosome is in a tightly supercoiled conformation. It is plausible that the growth defect of the rapA mutant on LB plates containing high concentrations of salt reflects the function of RapA in activation of transcription on tightly supercoiled DNA, as revealed in vitro. It is also possible that $\operatorname{rap} A$ is required for the maximal expression of some gene(s) important for cell growth in a high-salt environment. Recently, we found that the expression of the $\operatorname{rap} A$ gene is growth-phase-dependent, with its peak expression at the beginning of log-phase, and that the rap $A$ promoter is also growth rate-controlled (Cabrera and Jin 2001). However, further experiments are needed to dissect the role of rap $A$ in gene expression and cell growth.

\section{Materials and methods}

\section{Materials and reagents}

RNAP (Core RNAP and RNAP holoenzyme), 1:1 RNAP holoenzyme-RapA complex, and RapA (purified from 1:1 RNAP holoenzyme-RapA complex) were purified from E. coli MG1655 cells as described previously (Sukhodolets and Jin 1998). Nucleotides were purchased from Boehringer Mannheim, chemicals from Sigma, and ${ }^{32} \mathrm{P}-$-labeled nucleotides from Amersham. Restriction enzymes were from New England Biolabs. Supercoiled plasmid DNA used for transcription was of native superhelical density and was isolated from cultures grown in LB with $100 \mu \mathrm{g} / \mathrm{mL}$ of ampicillin. Plasmids were isolated using QIAGEN plasmid purification kits. Each of the plasmids used in the in vitro transcription assays contains a promoter followed by a terminator(s). The plasmid pRLG1617 containing the $\operatorname{rrnB}$ P1 promoter (Ross et al. 1990) was kindly provided by Wilma
Ross and Richard Gourse; pBHM332 containing the pyrBI promoter (Donahue and Turnbough 1990; Jin 1996) by Charles Turnbough, Jr.; and pCPG $\lambda \mathrm{t}_{\mathrm{R} 2}$ containing the phage $T 7$ A1 promoter (Reynolds et al. 1992) by Michael Chamberlin. The plasmid pDJ631 (ptac) was constructed by replacement of the EcoRI-HindIII fragment containing the $\operatorname{rrnB} P 1$ promoter in pRLG1617 with an EcoRI-HindIII-digested DNA fragment containing the tac promoter $(-38$ to +1$)$, which was generated by PCR using pKK223-3 (from Pharmacia Biotech) as a DNA template and the following two primers: 5'-ACAACAACAGAA TTCCTGTTGACAATTAATCATCGGCTCGTATAATG-3' and 5'-ACAACAACAAAGCTTCCACACATTATACGAGCCGA-3'.

\section{Construction of wild-type and mutant recombinant rapA clones}

The rapA clones used for overproduction of the recombinant RapA proteins were constructed as follows. For the wild-type rapA clone, the EcoRI-HindIII fragment from pDJ61 (Sukhodolets and Jin 2000) containing the rapA gene, in which the EcoRI site had been filled in with the Klenow fragment of DNA polymerase, was ligated into the BamHI and HindIII sites of the expression vector pQE31 (QIAGEN), in which the BamHI site had also been blunt-ended with Klenow enzyme. The resulting plasmid, pDJ3401, encoded a recombinant RapA protein containing the additional sequence MRGSHHHHHHTDQFT at its $\mathrm{N}$ terminus. The DNA fragment containing the entire wild-type rapA gene in pDJ3401was confirmed by sequencing. The rapA mutations in the SWI/SNF motifs Ia or II (Bork and Koonin 1993; Kolsto et al. 1993; Eisen et al. 1995) were constructed using the QuickChange site-directed mutagenesis kit (Stratagene) in accordance with the manufacturer's instructions. In either case, the wild-type rapA clone pDJ3401 was used as a DNA template. To make the SWI/SNF motif Ia mutation (Lys 183 to Ala), the mutagenic primers MS 21 (5'-CGA AGTGGGTTTAGGGGCAACCATTGAAGCCGGG-3') and MS 22 (5'-CCCGGCTTCAATGGTTGCCCCTAAACCCACTTCG $3^{\prime}$ ) were used. To make the SWI/SNF motif II mutation (Asp 280Glu 281 to Ala-Ala), the mutagenic primers MS23 (5'-GGACCT GCTGGTGGTCGCTGCAGCGCATCACCTGG-3') and MS24 (5'-CCAGGTGATGCGCTGCAGCGACCACCAGCAGGTCC$\left.3^{\prime}\right)$ were used. To ensure that no other mutation(s) in the rapA gene was generated during this process, a 1.2-kb EcoRI-EcoRV fragment containing the motif Ia or II mutation from the candidate plasmids was purified and used to replace the counterpart of the wild-type rapA clone (pDJ3401), resulting in pDJ3402 and pDJ3403, for the motif Ia and II rapA mutations, respectively. The entire EcoRI-EcoRV fragments containing the rapA mutation in motif Ia (pDJ3402) or II (pDJ3403) were confirmed by sequencing. All of the rapA clones were transformed into either XL1-Blue cells (Stratagene) or M15 (pRep4) cells (QIAGEN), and the transformants were selected on LB plates containing appropriate antibiotics.

\section{Purification of the recombinant RapA proteins}

M15 (pRep4) cells containing different recombinant rapA clones were grown at $37^{\circ} \mathrm{C}$ in $\mathrm{LB}$ medium supplemented with $100 \mu \mathrm{g} /$ $\mathrm{mL}$ ampicillin. At an $\mathrm{OD}_{600} \approx 0.6-0.8$ the expression of the protein was induced with 0.2 or $1 \mathrm{mM}$ IPTG. After induction for 1 $\mathrm{h}$, the cells were collected by centrifugation for $10 \mathrm{~min}$ at $5000 \mathrm{~g}$. About $1 \mathrm{~mL}$ of the cell pellet was resuspended in $\sim 30 \mathrm{~mL}$ of Extraction buffer $(50 \mathrm{mM}$ sodium phosphate, $0.3 \mathrm{M} \mathrm{NaCl}$ at $\mathrm{pH}$ 7.0) and lysed in a French Press. Following centrifugation for 30 
min at 20,000g, $1 \mathrm{~mL}$ of Talon metal affinity resin (Clontech) was added to the supernatant. After 4-5 washes with Extraction buffer containing $0.5 \mathrm{M} \mathrm{NaCl}$, the resin-bound protein was eluted with $200 \mathrm{mM}$ imidazole in Extraction buffer. The protein was dialyzed overnight against TGED buffer (10 mM Tris-HCl at $\mathrm{pH} 7.9,5 \%$ glycerol, $0.1 \mathrm{mM}$ EDTA, $0.1 \mathrm{mM}$ dithiothreitol) containing $50 \mathrm{mM} \mathrm{NaCl}$ and subjected to an additional purification step using either a Mono Q 5/10 (Pharmacia) or Resource $\mathrm{Q}$ column (Pharmacia) with an FPLC system (Pharmacia). A linear gradient from 0.05 to $0.5 \mathrm{M} \mathrm{NaCl}$ in $40 \mathrm{~mL}$ of TGED buffer was used to elute RapA. The recombinant proteins eluted from the column at $\sim 0.33-0.35 \mathrm{M} \mathrm{NaCl}$. The proteins were then concentrated to $\sim 1 \mathrm{mg} / \mathrm{mL}$ and transferred into protein storage buffer $(10 \mathrm{mM}$ Tris- $\mathrm{HCl}$ at $\mathrm{pH} 7.9,50 \%$ glycerol, $0.1 \mathrm{mM}$ EDTA, $0.1 \mathrm{mM}$ dithiothreitol and $0.1 \mathrm{M} \mathrm{NaCl}$ ) using Centriprep-30 concentrators (Amicon). In four independent repetitions of the above purification procedure, the yields were $0.5-1 \mathrm{mg}$ of recombinant RapA per $1 \mathrm{~mL}$ of pelleted cells.

\section{In vitro transcription assays}

A master reaction mixture was prepared as follows: $2 \mathrm{C}$ of $20 \times$ transcription buffer $(500 \mathrm{mM}$ Tris- $\mathrm{HCl}$ at $\mathrm{pH} 7.5,0.1 \mathrm{mM}$ EDTA, $0.2 \mathrm{mM}$ dithiothreitol, $2.5 \mathrm{mg} / \mathrm{mL}$ purified bovine serum albumin, and $50 \mathrm{mM}$ magnesium chloride), $2.1 \mu \mathrm{L}$ of plasmid DNA template (typically $0.2-1 \mu \mathrm{g} / \mu \mathrm{L}), 2.1 \mu \mathrm{L}$ of RNAP holoenzyme $(0.1 \mathrm{mg} / \mathrm{mL}), 2 \mu \mathrm{L}$ of purified RapA $(0.4-1 \mathrm{mg} / \mathrm{mL})$ or an equivalent amount of protein storage buffer, and $6.8 \mu \mathrm{L}$ of $\mathrm{H}_{2} \mathrm{O}$. Two-mictoliter aliquots of master reaction mixture (with or without RapA) were mixed with $2-\mu \mathrm{L}$ aliquots of stock solutions of $\mathrm{NaCl}, \mathrm{KCl}$, or potassium glutamate (pH adjusted to 7.5) to give the final salt concentrations (calculated for a final reaction volume of $5 \mu \mathrm{L}$ ) specified in figure legends. Following a 15 -min preincubation at $37^{\circ} \mathrm{C}$, the transcription reactions were initiated by the addition of $1 \mu \mathrm{L}$ of $5 \times$ NTP mix (the final NTP concentrations are indicated in figure legends) containing 30 $100 \mathrm{nCi}$ of either $\left[\alpha-{ }^{32} \mathrm{P}\right] \mathrm{CTP}$ or $\left[\alpha-{ }^{32} \mathrm{P}\right] \mathrm{UTP}$. Unless otherwise stated in figure legends, the reaction time was $30 \mathrm{~min}$ for multiple-round transcription assays. For single-round transcription reactions, the NTP mix also included heparin or competitor DNA. After incubation at $37^{\circ} \mathrm{C}$ for various lengths of time as indicated in figure legends, each $5-\mu \mathrm{L}$ transcription reaction was terminated by the addition of $2 \mu \mathrm{L}$ of Stop solution $(250 \mathrm{mM}$ EDTA at $\mathrm{pH} 8.0,50 \%$ glycerol, $0.05 \%$ xylene cyanol). Aliquots of the terminated reactions $(2.5-4 \mu \mathrm{L})$ were analyzed on $8 \%$ sequencing gels. Alternatively, the reactions were boiled for 2 min after the addition of Stop solution, with similar results. For kinetic studies, the master mix containing enzymes, DNA, and salt at fixed concentrations, as indicated in figure legends, was preincubated for $15 \mathrm{~min}$ at $37^{\circ} \mathrm{C}$; and the transcription reaction was then initiated by the addition of NTP mix as described above. Alternatively, the reaction was initiated with enzymes that were added to premixed DNA and NTPs (see figure legends). Aliquots were taken at specified timepoints, and transcription reactions were terminated and analyzed as described above. Gels were autoradiographed for 4-6 h (1× exposure) or $12-18 \mathrm{~h}$ ( $3 \times$ exposure) at $-80^{\circ} \mathrm{C}$ using BioMax MR film and BioMax MS screen (Kodak). Gels were also scanned on a PhosphorImager (Molecular Dynamics) to quantitate RNA transcripts.

\section{Other biochemical techniques}

The ATPase assays were performed as described previously (Sukhodolets and Jin 1998). RNAP-RapA binding assays for comparison of the affinities of the mutant and wild-type RapA proteins to RNAP were done using glycerol gradient ultracentrifugation as described previously (Sukhodolets and Jin 2000). Footprinting experiments were done essentially as described previously (Tugores and Brenner 1994).

\section{Bacterial strains and bacterial techniques}

The E. coli strains used were K12 MG1655 and its derivatives. The rapA null mutant, MG1655 rapA::cat (DJ473), was described previously (Sukhodolets and Jin 2000). Basic bacterial techniques were performed as described previously (Miller 1972). The wild-type and rapA mutant strains were grown in LB medium; a series of dilutions of overnight cultures were plated on LB plates containing different concentrations of $\mathrm{NaCl}$ (or sucrose), followed by incubation at $30^{\circ} \mathrm{C}$ for $48-96 \mathrm{~h}$. For the complementation test, the plasmid pDJ2635, which constitutively expresses RapA in the MG1655 background, was constructed as follows: an EcoRI-HindIII fragment from plasmid pDJ3401 containing the recombinant $r a p A$ gene was ligated into the EcoRI and HindIII sites of the pBR322 plasmid. Other rapA clones, which overproduced high levels of RapA either in the absence (pDJ3401) or presence (pDJ61) of inducer, affecting the growth of both wild-type strain and the rapA mutant, were not useful for the complementation test. To perform the complementation tests, the plasmids pDJ2635 and vector pBR322 were introduced into the wild-type strain and the rapA-null mutant strain by transformation. The cells harboring the plasmids were grown in media and plates supplemented with ampicillin (20 $\mu \mathrm{g} / \mathrm{mL}$ ), and their growth phenotypes were analyzed as described above.

\section{Acknowledgments}

We thank Drs. Debbie Hinton, Alicia Dombroski, and other colleagues for their comments on the manuscript.

The publication costs of this article were defrayed in part by payment of page charges. This article must therefore be hereby marked "advertisement" in accordance with 18 USC section 1734 solely to indicate this fact.

\section{References}

Adrian, M., ten Heggeler-Bordier, B., Wahli, W., Stasiak, A.Z., Stasiak, A., and Dubochet, J. 1990. Direct visualization of supercoiled DNA molecules in solution. EMBO J. 9: 45514554.

Altman, C.R., Solow-Cordero, D.E., and Chamberlin, M.J. 1994. RNA cleavage and chain elongation by Escherichia coli DNA-dependent RNA polymerase in a binary enzyme. RNA complex. Proc. Natl. Acad. Sci. 91: 3784-3788.

Andrews, B.J. and Herskowitz, I. 1989. Identification of a DNA binding factor involved in cell-cycle control of the yeast $\mathrm{HO}$ gene. Cell 57: 21-29.

Arndt, K.M. and Chamberlin, M.J. 1988. Transcription termination in Escherichia coli. Measurement of the rate of enzyme release from Rho-independent terminators. I. Mol. Biol. 202: 271-285.

Bednar, J., Furrer, P., Stasiak, A., Dubochet, J., Egelman, E.H., and Bates, A.D. 1994. The twist, writhe and overall shape of supercoiled DNA changes during counterion-induced transition from a loosely to a tightly interwound superhelix. Possible implications for DNA structure in vivo. J. Mol. Biol. 235: 825-847.

Bork, P. and Koonin, E.V. 1993. An expanding family of heli- 
cases within the 'DEAD/H' superfamily. Nucleic Acids Res. 21: 751-752.

Borukhov, S., Sagitov, V., and Goldfarb, A. 1993. Transcript cleavage factors from E. coli. Cell 72: 459-466.

Brady, G.W., Fein, D.B., Lambertson, H., Grassian, V., Foos, D., and Benham, C.J. 1983. X-ray scattering from the superhelix in circular DNA. Proc. Nat1. Acad. Sci. 80: 741-744.

Burgess, R.R., Travers, A.A., Dunn, J.J., and Bautz, E.K. 1969. Factor stimulating transcription by RNA polymerase. $\mathrm{Na}$ ture 221: 43-46.

Burgess, R.R., Erickson, B., Gentry, D., Gribskov, M., Hager, D., Lesley, S., Strickland, M., and Thompson, N. 1987. Bacterial RNA polymerase subunits and genes. In RNA polymerase and the regulation of transcription (eds. W.S. Reznikoff et al.), pp. 3-15. Elsevier Science Publishing, New York, NY.

Cabrera, J. and Jin, D.J. 2001. Growth phase and growth rate regulation of the rapA gene encoding the RNA polymeraseassociated protein RapA in Escherichia coli. J. Bacteriol. 183: 6126-6134

Carlson, M., Osmond, B.C., Neigeborn, L., and Botstein, D. 1984. A suppressor of SNF1 mutations causes constitutive high-level invertase synthesis in yeast. Genetics 107: 19-32.

Cayley, S., Lewis, B.A., Guttman, H.J., and Record, Jr., M.T. 1991. Characterization of the cytoplasm of Escherichia coli $\mathrm{K}-12$ as a function of external osmolarity. Implications for protein-DNA interactions in vivo. J. Mol. Biol. 222: 281-300.

Donahue, J.P. and Turnbough, Jr., C.L. 1990. Characterization of transcriptional initiation from promoters $\mathrm{P} 1$ and $\mathrm{P} 2$ of the pyrBI operon of Escherichia coli K12. I. Biol. Chem. 265: 19091-19099.

Eisen, J.A., Sweder, K.S., and Hanawalt, P.C. 1995. Evolution of the SNF2 family of proteins: Subfamilies with distinct sequences and functions. Nucleic Acids Res. 23: 2715-2723.

Feng, G.H., Lee, D.N., Wang, D., Chan, C.L., and Landick, R. 1994. GreA-induced transcript cleavage in transcription complexes containing Escherichia coli RNA polymerase is controlled by multiple factors, including nascent transcript location and structure. J. Biol. Chem. 269: 22282-22294.

Friedman, D.I. and Gottesman, M. 1983. Lytic mode of lambda development. In Lambda II (eds. R.W. Hendrix, J.W. Roberts, F.W. Stahl, and R.A. Weisberg), pp. 21-51. Cold Spring Harbor Laboratory Press. Cold Spring Harbor, NY.

Gavin, I., Horn, P.J., and Peterson, C.L. 2001. SWI/SNF chromatin remodeling requires changes in DNA topology. Mol. Cell 7: 97-104.

Gourse, R.L. 1988. Visualization and quantitative analysis of complex formation between $E$. coli RNA polymerase and an rRNA promoter in vitro. Nucleic Acids Res. 16: 9789-9809.

Greenblatt, J. and Li, J. 1981. Interaction of the sigma factor and the nus $A$ gene protein of $E$. coli with RNA polymerase in the initiation-termination cycle of transcription. Cell 24: 421428.

Havas, K., Flaus, A., Phelan, M., Kingston, R., Wade, P.A., Lilley, D.M., and Owen-Hughes, T. 2000. Generation of superhelical torsion by ATP-dependent chromatin remodeling activities. Cell. 103: 1133-1142.

Hsu, L.M., Vo, N.V., and Chamberlin, M.J. 1995. Escherichia coli transcript cleavage factors GreA and GreB stimulate promoter escape and gene expression in vivo and in vitro. Proc. Natl. Acad. Sci. 92: 11588-11592.

Jin, D.J. 1996. A mutant RNA polymerase reveals a kinetic mechanism for the switch between nonproductive stuttering synthesis and productive initiation during promoter clearance. J. Biol. Chem. 271: 11659-11667.

Juang, Y.L. and Helmann, J.D. 1994. The delta subunit of Bacillus subtilis RNA polymerase. An allosteric effector of the initiation and core-recycling phases of transcription. J. Mol. Biol. 239: 1-14.

Kolsto, A.B., Bork, P., Kvaloy, K., Lindback, T., Gronstadt, A., Kristensen, T., and Sander, C. 1993. Prokaryotic members of a new family of putative helicases with similarity to transcription activator SNF2. J. Mol. Biol. 230: 684-648.

Lewis, L.K., Jenkins, M.E., and Mount, D.W. 1992. Isolation of DNA damage-inducible promoters in Escherichia coli: Regulation of $\operatorname{polB}(\operatorname{din} A), \operatorname{din} G$, and $\operatorname{din} H$ by LexA repressor. $J$. Bacteriol. 174: 3377-3385.

Miller, J.H. 1972. Experiments in molecular genetics. Cold Spring Harbor Laboratory Press. Cold Spring Harbor, NY.

Minakhin, L., Bhagat, S., Brunning, A., Campbell, E.A., Darst, S.A., Ebright, R.H., and Severninov, K. 2001. Bacterial RNA polymerase subunit omega and eukaryotic RNA polymerase subunit RPB6 are sequence, structural and functional homologs and promote RNA polymerase assembly. Proc. Nat1. Acad. Sci. 98: 392-397.

Muchardt, C. and Yaniv, M. 1999. ATP-dependent chromatin remodelling: SWI/SNF and Co. are on the job. J. Mol. Biol. 293: $187-198$.

Mukherjee, K. and Chatterii, D. 1997. Studies on the omega subunit of Escherichia coli RNA polymerase and its role in the recovery of denatured enzyme activity. Eur. J. Biochem. 247: 884-889.

Muzzin, O., Campbell, E.A., Xia, L., Severinova, E., Darst, S.A., and Severinov, K. 1998. Disruption of Escherichia coli hepA, an RNA polymerase-associated protein, causes UV sensitivity. J. Biol. Chem. 273: 15157-15161

Pazin, M.J. and Kadonaga, J.T. 1997. SWI2/SNF2 and related proteins: ATP-driven motors that disrupt protein-DNA interactions? Cell 88: 737-740.

Peterson, C.L. 1996. Multiple SWItches to turn on chromatin? Curr. Opin. Genet. Dev. 6: 171-175.

Peterson, C.L. and Tamkun, J.W. 1995. The SWI-SNF complex: A chromatin remodeling machine? Trends Biochem. Sci. 20: $143-146$.

Reynolds, R., Bermudez-Cruz, R.M., and Chamberlin, M.J. 1992. Parameters affecting transcription termination by Escherichia coli RNA polymerase. I. Analysis of 13 rho-independent terminators. J. Mol. Biol. 224: 31-51.

Richey, B., Cayley, D.S., Mossing, M.C., Kolka, C., Anderson, C.F, Farrar, T.C., and Record, Jr., M.T. 1987. Variability of the intracellular ionic environment of Escherichia coli. Differences between in vitro and in vivo effects of ion concentrations on protein-DNA interactions and gene expression. J. Biol. Chem. 262: 7157-7164.

Ristic, D., Wyman, C., Paulusma, C., and Kanaar, R. 2001. The architecture of the human Rad54-DNA complex provides evidence for protein translocation along DNA. Proc. Nat1. Acad. Sci. 98: 8454-8460.

Ross, W., Thompson, J.F., Newlands, J.T., and Gourse, R.L. 1990. E. coli Fis protein activates ribosomal RNA transcription in vitro and in vivo. EMBO J. 9: 3733-3742.

Rybenkov, V.V., Vologodskii, A.V., and Cozzarelli, N.R. 1997a. The effect of ionic conditions on the conformations of supercoiled DNA. I. Sedimentation analysis. J. Mol. Biol. 267: 299-311.

Rybenkov, V.V., Vologodskii, A.V., and Cozzarelli, N.R. 1997b. The effect of ionic conditions on the conformations of supercoiled DNA. II. Equilibrium catenation. J. Mol. Biol. 267: 312-323.

Sparkowski, J. and Das, A. 1991. Location of a new gene, greA, on the Escherichia coli chromosome. J. Bacteriol. 173: 52565257.

Spengler, S.J., Stasiak, A., and Cozzarelli, N.R. 1985. The ste- 
reostructure of knots and catenanes produced by phage lambda integrative recombination: Implications for mechanism and DNA structure. Cell 42: 325-334.

Sukhodolets, M.V. and Jin, D.J. 1998. RapA, a novel RNA polymerase-associated protein, is a bacterial homolog of SWI2/ SNF2. J. Biol. Chem. 273: 7018-7023.

. 2000. Interaction between RNA polymerase and RapA, a bacterial homolog of the SWI/SNF protein family. J. Biol. Chem. 275: 22090-22097.

ten Heggeler-Bordier, B., Wahli, W., Adrian, M., Stasiak, A., and Dubochet, J. 1992. The apical localization of transcribing RNA polymerases on supercoiled DNA prevents their rotation around the template. EMBO J. 11: 667-672.

Tugores, A. and Brenner, D.A. 1994. A method for in vitro DNase I footprinting analysis on supercoiled templates. BioTechniques 17: 410-412.

Zhou, Y.N. and Jin, D.J. 1998. The rpoB mutants destabilizing initiation complexes at stringently controlled promoters behave like 'stringent' RNA polymerases in Escherichia coli. Proc. Natl. Acad. Sci. 95: 2908-2913. 


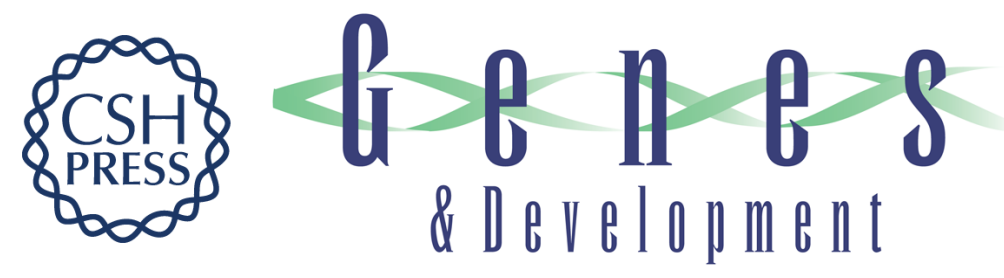

\section{RapA, a bacterial homolog of SWI2/SNF2, stimulates RNA polymerase recycling in transcription}

Maxim V. Sukhodolets, Julio E. Cabrera, Huijun Zhi, et al.

Genes Dev. 2001, 15:

Access the most recent version at doi:10.1101/gad.936701

$\begin{array}{ll}\text { References } & \begin{array}{l}\text { This article cites } 44 \text { articles, } 17 \text { of which can be accessed free at: } \\ \text { http://genesdev.cshlp.org/content/15/24/3330.full.html\#ref-list-1 }\end{array}\end{array}$

License

Email Alerting

Receive free email alerts when new articles cite this article - sign up in the box at the top Service right corner of the article or click here.

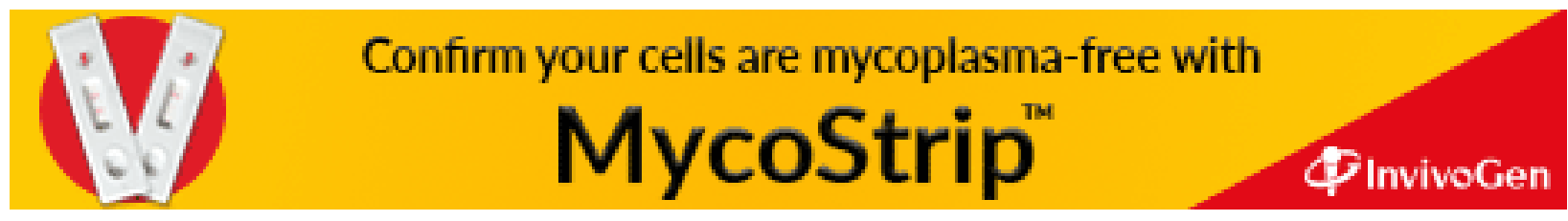

